\title{
Dynamics in Canonical Spin Glasses Observed by Muon Spin Depolarization
}

\author{
I. A. Campbell \\ Physique des Solides, Université Paris Sud, 91405 Orsay, France \\ A. Amato, F. N. Gygax, D. Herlach, and A. Schenck \\ Paul Scherrer Institute and Swiss Federal Institute of Technology (ETH) Zürich, \\ CH5232 Villigen PSI, Switzerland \\ R. Cywinski and S. H. Kilcoyne* \\ J.J. Thomson Laboratory, University of Reading, Reading RG6 2AF, United Kingdom
}

(Received 23 September 1993)

\begin{abstract}
Muon spin depolarization has been studied in moderately concentrated $A g \mathrm{Mn}$ and $A u \mathrm{Fe}$ alloys from the freezing temperature $T_{g}$ up to $300 \mathrm{~K}$. The muon depolarization function can be analyzed to show that the temperature dependence of the strongly nonexponential form of the local spin autocorrelation function in these canonical alloys is similar to that observed in numerical simulations on Ising spin glasses. The dynamic behavior above $T_{g}$ appears to be an intrinsic precursor to spin glass freezing.
\end{abstract}

PACS numbers: $75.50 .2 k, 76.75 .+i$

It has been known for a long time that the dynamics of glassy systems is not simple and there is still no overall explanation of their relaxation behavior. Experimentally, spin glasses (SGs) below the ordering temperature $T_{g}$ show slow quasilogarithmic relaxation [1]; the dynamics has been studied in detail and the behavior has been ascribed to a complex phase space structure [2]. For temperatures above $T_{g}$, the relaxation is much faster but is still strongly nonexponential, at least close to $T_{g}$ [3]. For the $\pm J$ Ising SG, numerical simulations [4] have shown a relaxation of the autocorrelation function of the form

$$
q(t) \approx t^{-x} \exp \left[-(t / \tau)^{\beta}\right],
$$

with the stretched exponential exponent $\beta$ having a value of about $\frac{1}{3}$ at $T_{g}$ and attaining the simple exponential value of 1 as in a conventional paramagnet only for $T$ about 4 times $T_{g}$. It has been conjectured that the SG transition is analogous to a percolation transition in phase space so that this type of dynamics could be characteristic of all SGs [5]; further simulations on other Ising and $X Y$ SGs indeed show similar behavior [5]. It is important to know if the dynamics of real SGs shows the same pattern. We have used the muon spin depolarization technique to investigate local moment relaxation in metallic SGs.

Muon spin depolarization (usually referred to as $\mu \mathrm{SR}$ ) is a very effective probe of local moment dynamics and SGs have been studied by a number of groups using this technique [6]. In the temperature regime above the ordering temperature the muons, initially polarized, are gradually depolarized by the fluctuating fields coming from neighboring local moments; the faster these moments relax, the more slowly the muons are depolarized - the familiar motional narrowing effect. We have addressed anew the question of the local spin dynamics above $T_{g}$ in canonical noble metal based SGs, using systems with weak host nuclear moments ( $A g \mathrm{Mn}$ and $A u \mathrm{Fe}$ ) and choosing fairly high impurity concentration samples. After initial experiments at the Rutherford Appleton
Laboratory we have made measurements at the Paul Scherrer Institute in zero field and in longitudinal fields of 0.1 and $0.6 \mathrm{~T}$ with an experimental setup which can cover a range of muon polarization decay times from about $10 \mathrm{~ns}$ to about $50 \mu \mathrm{s}$. The data show that there is a wide distribution of local moment relaxation rates at temperatures near $T_{g}$; right at $T_{g}$ the broad relaxation time distribution takes up a form which may well be universal for all SGs. The distribution narrows with increasing temperature until the relaxation becomes "simple" (a unique relaxation time for all the local moments) above about 4 times $T_{g}$.

We also obtain information on muon diffusion and trapping in these alloys.

We studied $A g \mathrm{Mn}$ samples with 5, 7, and 10 at. $\% \mathrm{Mn}$, and a $A u(5 \%)$ Fe sample. The samples were prepared by melting and quenching a number of times; they were then severely cold worked and given a final anneal and quench. From the known metallurgy of these systems the impurity concentration should be uniform throughout the sample after this treatment. The $T_{g}$ values of the samples are $20,25,32$, and $26 \mathrm{~K}$, respectively. Both $\mathrm{Ag}$ and $\mathrm{Au}$ have small nuclear moments (in contrast to $\mathrm{Cu}$ ) so we can ignore the depolarization of the muon spins by the host nuclei; all depolarization will be due to the interactions with the $\mathrm{Mn}$ or Fe local moments.

The muon interaction with a local moment is dominantly of the dipole-dipole type,

$$
H=A\left[\mathbf{S} \cdot \mathbf{I}-3(\mathbf{S} \cdot \mathbf{r})(\mathbf{I} \cdot \mathbf{r}) / r^{2}\right] .
$$

If the local moment is relaxing exponentially at a rate $1 / \tau$ then the muon depolarization rate $\lambda$ is proportional to $A^{2} \tau$. Earlier SG $\mu \mathrm{SR}$ data above $T_{g}$ were analyzed assuming a unique relaxation time $\tau$ for all the local spins at each temperature, together with a distribution of $A$ values due to the random positions of the muons in the alloy. For nondiffusing muons in the limit of very dilute alloy these assumptions lead to an average muon polarization decay of the "root exponential" form 

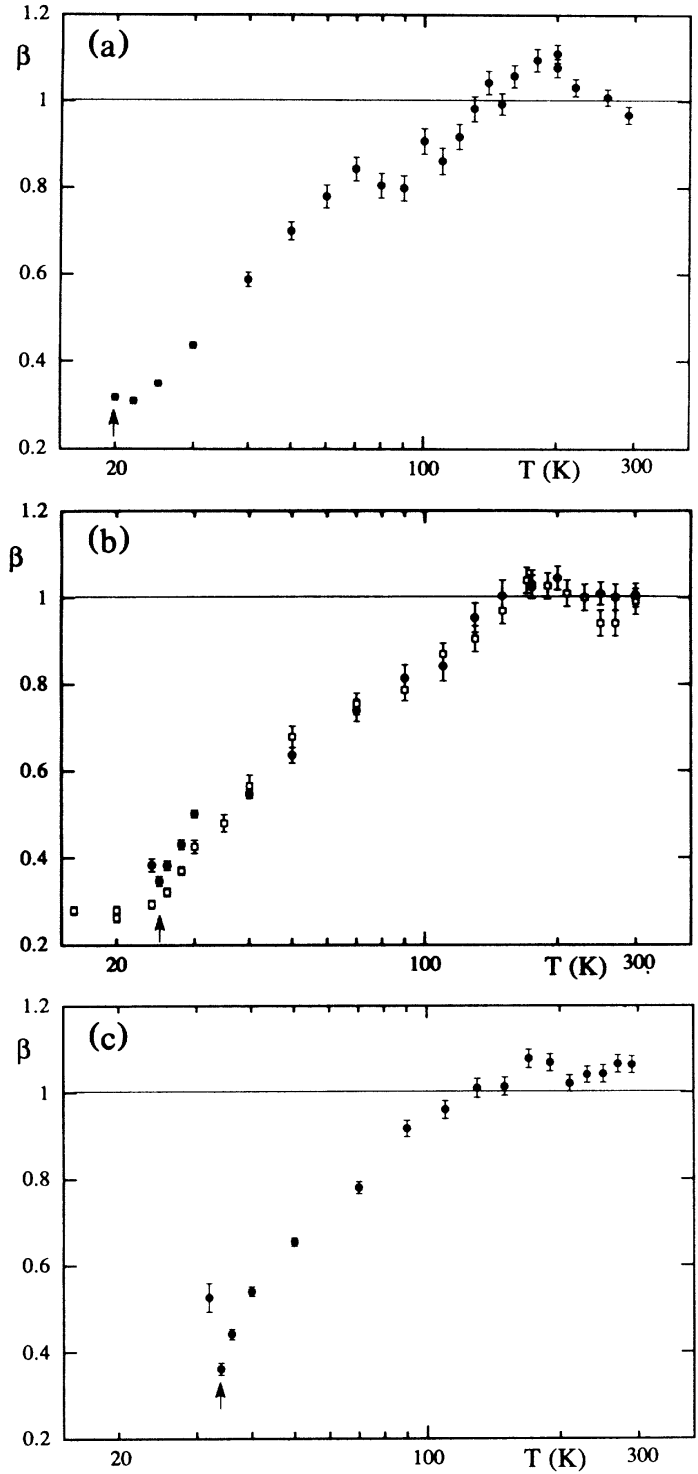

FIG. 1. The stretched exponential exponent $\beta$ as a function of temperature for zero field (ZF) data on (a) $\mathrm{AgMn} 5$ at. \%,

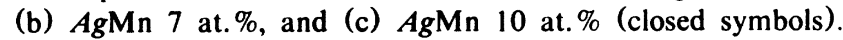
The arrows indicate the $T_{g}$ values of each sample. Just below $T_{g}$ the apparent value of $\beta$ in ZF increases sharply as can be seen for the lowest temperature point in plot (c); this is an artifact as the stretched exponential is an inappropriate fit function below $T_{g}$ [6]. Above $T_{g}$ the longitudinal field (LF) data are essentially identical to the ZF data, while below $T_{g}$ they are quite different and can be fitted with a constant $\beta$ of about 0.3 [the open squares of (b) are 0.6 T LF data].

$$
P(t)=\exp \left[-(\lambda t)^{\beta}\right]
$$

with $\beta=\frac{1}{2}$ and $\lambda$ proportional to $\tau$. We have chosen to work with rather concentrated alloys where almost every muon site will have at least one local moment nearby, so the geometrical distribution of $A$ values will be considerably narrower than in the dilute limit. If $\tau$ were unique at each temperature, one would have a $P(t)$ whose shape was temperature independent and close to exponential, 1292

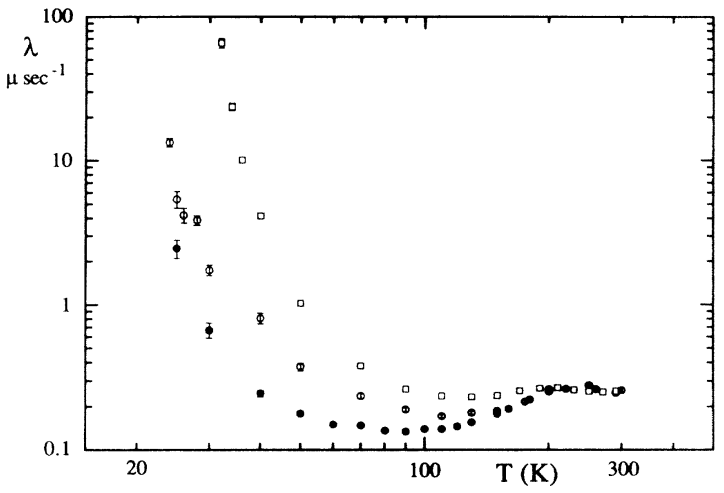

FIG. 2. The temperature dependence of the muon depolarization rate $\lambda$ as defined in Eq. (3) for $\mathrm{ZF}$ data on the three AgMn samples (10\% sample: squares; $7 \%$ sample: open circles; $5 \%$ sample: closed circles). $\lambda$ is in $\mu \mathrm{s}^{-1}$. LF values are again essentially identical above $T_{g}$.

i.e., with an effective $\beta$ close to, but rather smaller than, 1.

In the experiments, the initial muon asymmetry was measured for a pure Ag sample of the same form as the alloy samples, in the longitudinal (or zero) field geometry but in the presence of a small transverse field. The asymmetry value $a_{0}$ was then held fixed throughout the fitting procedure on the alloy data. As to be expected from earlier muon experiments on SGs [6], the longitudinal field produced a major change in the depolarization pattern for temperatures below $T_{g}$ (corresponding to the presence of static spin moments), but had very little effect for the purely dynamic regime above $T_{g}$ [see Fig. 1(b)]. From the usual $\chi^{2}$ criterion we find at each temperature a statistically excellent fit to the stretched exponential form for $P(t)$, Eq. (1). $\lambda(T)$ tends to diverge as $T$ tends to $T_{g}$, with $\beta(T)$ which varies strongly from near $\frac{1}{3}$ at $T_{g}$ to near 1 at higher temperatures (Fig. 1, 2, and 3). As we will show below, for most of the temperature range the form of $P(t)$ is controlled by an intrinsic distribution of the spin relaxation times rather than by the geometrical $A$ distribution effect.

Before further considering the local moment dynamics, we will make a necessary digression to discuss the muon diffusion. Results on very dilute $\mathrm{Ag}$ and $\mathrm{Au}$ alloys with rare earth impurities $[7,8]$ indicated that the muon does not diffuse until about $190 \mathrm{~K}$ in $\mathrm{Ag}$ and about $100 \mathrm{~K}$ in Au. In our $A g M n$ alloys we would expect the local moment relaxation time to decrease steadily with $T$ (Korringa mechanism), so in the absence of muon diffusion $\lambda(T)$ should drop continuously as $T$ increases. Instead we observe for each sample an initial drop in $\lambda(T)$ followed by an increase to a broad maximum at about $250 \mathrm{~K}$, with $\lambda(T)$ becoming concentration independent in this temperature range. We can ascribe this to muon diffusion and trapping, with the following scenario. Above some critical temperature the muons begin to diffuse, and the increase in $\lambda(T)$ must correspond to the muon diffusing to a trapping site next to a $\mathrm{Mn}$ atom, where $A$ is stronger 


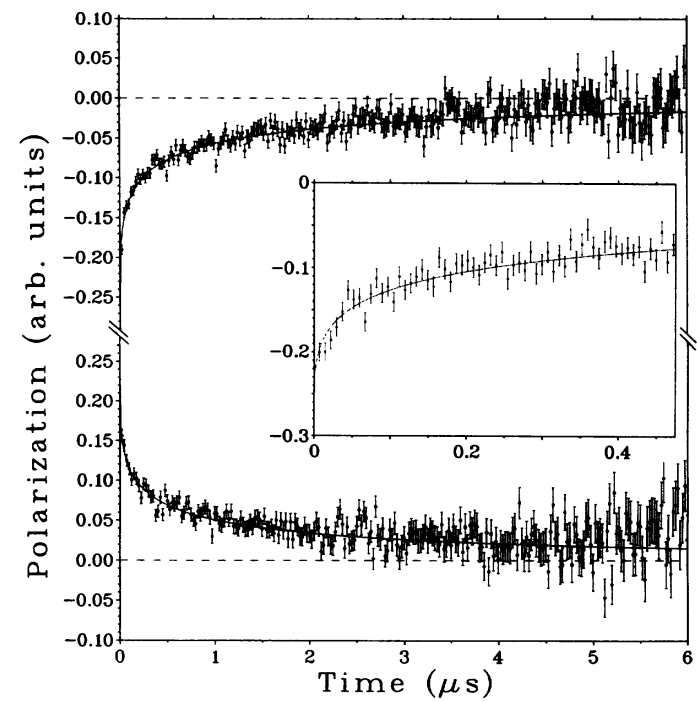

FIG. 3. An example of raw muon depolarization data as a function of time obtained in $\mathrm{AgMn} 7 \%$ at $T=26 \mathrm{~K}$ (just above $T_{g}=25 \mathrm{~K}$ ) in a longitudinal field of $0.6 \mathrm{~T}$. The upper part/lower part show data from a forward/backward counter telescope: Initial asymmetries -0.27 and +0.24 , respectively, and fit parameters $\beta=0.32(1)$ and $\lambda=4.0(6) \mu \mathrm{s}^{-1}$. The inset shows a blown up early part of the forward time spectrum.

than the average effective $A$ value felt by the muon at its initial random position in the alloy. For a certain range of temperature $\lambda(T)$ thus increases as the diffusion becomes faster. Once all muons are trapped they are all in similar positions at a trapping site next to a $\mathrm{Mn}$ atom whatever the $\mathrm{Mn}$ concentration, so $\lambda(T)$ becomes concentration independent when the temperature is high enough (Fig. 2). At a given temperature in the temperature range where the muon diffusion time to a trapping site is of the order of a microsecond, the initial depolarization rate at short times (when the muon is still in a random position) is slower than the depolarization rate at long times (where the muon has snuggled up to a $\mathrm{Mn}$ and so feels a larger $A$ ); when the data are analyzed this leads to an effective exponent $\beta$ which is larger than 1. This can be observed in Fig. 1 for the temperature range between 150 and $300 \mathrm{~K}$. We can note that muon diffusion effects appear in $\lambda(T)$ starting at a lower temperature than in Refs. [7,8]; this is because in the present concentrated alloys a jump of a single lattice spacing will change the muon environment significantly, whereas in the dilute alloys a long diffusion path is needed to bring the muon near a magnetic site. In our alloys the form of $\lambda(T)$ indicates that the diffusion effects begin to be significant above about $120 \mathrm{~K}$.

If measurements were continued above $300 \mathrm{~K}$ we would expect $\lambda(T)$ to start decreasing again regularly with increasing temperature and $\beta(T)$ to go to 1 . The $A u \mathrm{Fe}$ data (which are not given here) show behavior which is similar to that observed in the AgMn alloys, but with a more complicated temperature dependence corre- sponding to multiple trapping sites.

Thus for temperatures above about $120 \mathrm{~K}$ the $\mathrm{AgMn}$ data are complicated by the effects of the muon diffusion. Below this temperature we can consider the muon as static and occupying a random position in the alloy. From now on we will discuss the information on the spin dynamics which we can obtain from the data in this lower temperature range. For each alloy $\lambda(T)$ increases regularly with decreasing $T$ because of the critical slowing down of the $\mathrm{Mn}$ relaxation. If there were a unique relaxation time $\tau$ for all the Mn spins at each $T$, because of the geometrical distribution of $A, \beta(T)$ should be somewhat less than 1 and should be independent of $T$ as we have seen above. This is not the case. What we observe is that for each sample, $\beta(T)$ is essentially equal to $\frac{1}{3}$ at $T_{g}$ and then increases strongly with increasing $T$. For the $5 \% \mathrm{Mn}$ sample $\beta(T)$ reaches a plateau value of about 0.8 at temperatures from about $70 \mathrm{~K}$ until muon diffusion effects set in at about $120 \mathrm{~K}$. In the higher concentration samples the plateau values are rather higher and are blurred by the muon diffusion effects but appear to set in at a temperature of about 4 times $T_{g}$ in each case. We associate the plateau regime with a situation where the relaxation time is unique and the muon depolarization rate distribution is slightly nonexponential because of the geometrical $\boldsymbol{A}$ distribution effect; at lower temperatures the strong reduction in $\beta(T)$ must be due to a distribution in the spin relaxation times $\tau$. These results show clearly that a unique value of $\tau$ for all $\mathrm{Mn}$ spins is a good approximation only for $T$ greater than about $4 T_{g}$. Below this temperature, the relaxation rate distribution becomes progressively wider; very close to $T_{g} \lambda(T)$ diverges and the relaxation rate distribution takes up a standard form for all samples (including the $A u \mathrm{Fe}$ sample) resulting in the observed shape of the depolarization function:

$$
P(t)=\exp \left[-(\lambda t)^{1 / 3}\right] \text {. }
$$

The same limiting behavior with a stretched exponential having exponent close to $\frac{1}{3}$ as $T$ tends to $T_{g}$ has been observed recently in two other quite different spin glasses, $\mathrm{Y}\left(\mathrm{Mn}_{1-x} \mathrm{Al}_{x}\right)_{2}$ and $\mathrm{La}_{2} \mathrm{Cu}_{0.25} \mathrm{Co}_{0.75} \mathrm{O}_{4+\delta}[9,10]$, which suggests that this form may well be universal for all spin glasses at the freezing temperature.

It would be most instructive if we could derive the form of the spin autocorrelation relaxation function $q(t)$ directly from the shape of the muon $P(t)$. We can discuss the results by making the approximation that the overall decay of the autocorrelation function is given by the sum of individual exponential relaxations with a weighting for the probability distribution of $\tau$. The approximation by a sum of relaxation times is close to the model of independently relaxing clusters which has been used to analyze Mössbauer data [11] and to discuss critical phenomena [12]. If we assume that each muon $i$ has an exponential depolarization rate $\lambda_{i}$ then the form of the overall $P(t)$ reflects the distribution of $\lambda_{i}$ through a Laplace transformation. Take as an example $P(t)$ 


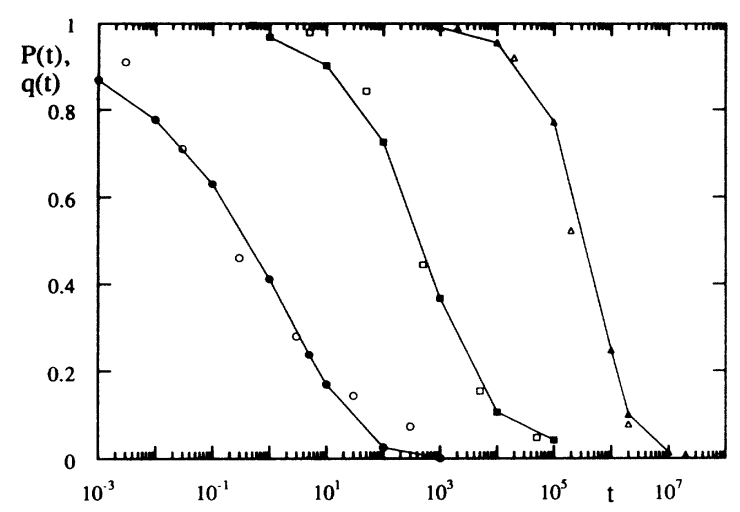

FIG. 4. A comparison of stretched exponential " $P(t)$ " curves $\left(\beta=\frac{1}{3}, \frac{1}{2}\right.$, and $\frac{2}{3}$ from left to right, full symbols) and the corresponding Laplace transform " $q(t)$ " curves (open symbols); see text. For $\beta=\frac{1}{2}$ the transform is analytic; for the others it is numerical. The curves have been shifted by arbitrary factors along the $t$ axis as only their relative shapes are significant.

$=\exp \left[-(\lambda t)^{1 / 2}\right]$, the only stretched exponential for which the handbooks give an analytic Laplace transformation. For this $P(t)$, the distribution of $\lambda_{i}$ values is given by

$$
G\left(\lambda_{i}\right)=\left[\lambda^{2} / 2\left(\pi \lambda_{i}^{3}\right)^{1 / 2}\right] \exp \left(-\lambda^{4} / 4 \lambda_{i}\right) .
$$

At the same level of approximation, the depolarization of each muon $i$ reflects the relaxation of a local spin with a relaxation time $\tau_{i}$ proportional to $\lambda_{i}$. The relaxation of the spin autocorrelation function $q(t)$ will then be given by

$$
\int_{0}^{\infty} G\left(\lambda_{i}\right) \exp \left(-t / \alpha \lambda_{i}\right) d \lambda_{i}
$$

where $\tau_{i}=\alpha \lambda_{i}$.

If we calculate this we find

$$
q(t)=\left(1+4 t / \alpha \lambda^{4}\right)^{-1 / 2} \text {. }
$$

We deduce a strongly nonexponential relaxation for the spin correlation function $q(t)$ from the nonexponential $P(t)$, but the shape of the derived function is not identical to that of $P(t)$. The precise form obtained for $q(t)$ should be taken with considerable caution as there is no reason to believe that the assumptions we have made are exact; the true form of the $P(t)$ curve may not be strictly a pure stretched exponential, the relaxation of each individual local spin may not be in fact purely exponential, and more than one spin will contribute to the depolarization of a given muon. However, if despite these caveats we take the calculated form for $q(t)$ as it stands and compare it to $\exp \left(-t^{1 / 2}\right)$ (Fig. 4), we find the two curves are quite similar and that they correspond to similar relaxation time widths. This means that with the interpretation we have used, to a $P(t)$ of the stretched exponential form with $\beta=\frac{1}{2}$ there corresponds to a good approximation a $q(t)$ with similar form and similar exponent. On the strength of this (and from numerical integrations at other values of $\beta$; Fig. 4), we can deduce that if we were to measure $q(t)$ directly at each temperature $T$ it would be of similar form to $P(t)$, i.e., close to a stretched exponential with the same $\beta(T)$ as we observe in the $P(t)$ measurement at $T$. (However, the relationship between the $\mu \mathrm{SR} \lambda$ and the spin correlation time depends on $\beta$ at each temperature, which may explain the apparent inconsistency between $\mu$ SR estimates of the SG dynamic critical exponents and values obtained from direct magnetic measurements [6].) We can then make the following statement: The local spin relaxation rate distribution has essentially a unique value $1 / \tau$ for temperatures above about 4 times $T_{g}$. It then tends to widen as the temperature is lowered; when $T_{g}$ is reached the spin relaxation rates are broadly distributed and the form of this distribution [which controls the shape of $P(t)$ ] appears to be universal in the alloys we have studied and possibly more generally in all spin glasses. At $T_{g}$ the global local spin relaxation function is close to the form of Eq. (4).

In conclusion, the data we have obtained provide clear evidence that in real spin glasses as in the simulated Ising spin glass system, there is an upper characteristic temperature, well above $T_{g}$, below which the global local spin relaxation function $q(t)$ begins to be nonexponential (with a distribution of local spin relaxation rates). The form of $q(t)$ continues to widen as the temperature is lowered until at $T_{g}, q(t)$ is close to the form of a stretched exponential with exponent $\beta=\frac{1}{3}$. This pattern of dynamic behavior in the temperature regime down to the freezing temperature seems to be a necessary and intrinsic part of the spin glass ordering process.

*Present address: Rutherford Appleton Laboratory, Chilton, Didcot OX11 0QX, United Kingdom.

[1] R. Omari, J. J. Préjean, and J. Souletie, J. Phys. (Paris) 45, 1809 (1984).

[2] F. Lefloch, J. Hammann, M. Ocio, and E. Vincent, Europhys. Lett. 18, 647 (1992).

[3] F. Mezei and A. P. Murani, J. Magn. Magn. Mater. 14, 211 (1979).

[4] A. T. Ogielski, Phys. Rev. B 32, 7384 (1985).

[5] I. A. Campbell, J. M. Flesselles, R. Jullien, and R. Botet, J. Phys. C 20, L47 (1987); L. Bernardi and I. A. Campbell (unpublished).

[6] Y. J. Uemura et al., Phys. Rev. B 31, 546 (1985); R. H. Heffner et al., J. Appl. Phys. 53, 2174 (1982); H. Pinkvos, A. Kalk, and C. Schwink, Phys. Rev. B 41, 590 (1990).

[7] J. A. Brown et al., Phys. Rev. Lett. 47, 261 (1981).

[8] M. E. Schillaci et al., in Electronic Structure and Properties of Hydrogen in Metals, edited by P. Jena and C. B. Satterthwaite (Plenum, New York, 1983), p. 507.

[9] R. Cywinski and B. D. Rainford (to be published):

[10] A. Lappas, K. Prassides, A. Amato, F. N. Gygax, and A. Schenk (to be published)

[11] C. Meyer, F. Hartmann-Boutron, J. M. Greneche, and F. Varret, J. Phys. (Paris), Colloq. 49, C8-1155 (1988); A. Pösinger et al., J. Phys. Condens. Matter 3, 2713 (1991).

[12] M. A. Continentino and A. P. Malozemoff, Phys. Rev. B 34, 471 (1986). 